\title{
Correction to "Integrative Analysis of Transcriptomics, Proteomics, and Metabolomics Data of White Adipose and Liver Tissue of High- Fat Diet and Rosiglitazone-Treated Insulin-Resistant Mice Identified Pathway Alterations and Molecular Hubs"
}

\author{
David Meierhofer, Christopher Weidner, and Sascha Sauer* \\ J. Proteome Res. 2014, 13 (12), 5592-5602. DOI: 10.1021/pr5005828 \\ $\mathrm{B}$ ecause several related errors during the preparation of the \\ B manuscript, the description of in the "Network Analysis" \\ section led to confusion. Moreover, we encountered errors in

\section{LAST TWO PARAGRAPHS OF INTEGRATING METABOLOMICS DATA SECTION} \\ TRANSCRIPTOMICS, PROTEOMICS, AND
} the assignments of low- and high-fat diet (LFD, HFD) and ambiguities in the section termed "Integrating Transcriptomics, Proteomics, and Metabolomics Data” that also might lead to potential incomprehensiveness.

Importantly, the main results and conclusions are not affected by the clarifying changes made here. We sincerely apologize for these regrettable mistakes and for any confusion this may have caused.

For better overview, we here provide the corrected errors in the context of the affected text sections.

\section{NETWORK ANALYSIS}

Using our protein expression data sets, we analyzed protein networks to infer and characterize hub proteins that may exert major functions during HFD and drug treatment with RGZ. Only the proteins which were down regulated in HFD and up regulated in RGZ-HFD in WAT generated large networks (Figure 5), in contrast to liver tissue. The KEGG pathway affiliation of these networks was examined and revealed that the networks belong to energy metabolism (e.g., OXPHOS, TCA cycle, and branched chain amino acids (BCAA)). The network similarity between LFD/HFD and RGZ-HFD/HFD in WAT (Figure 5) shows that the drug treatment by RGZ puts the HFD condition in part back to a LFD-like condition. This was confirmed by a direct network comparison of LFD versus RGZHFD, resulting in a neutralization of the TCA and OXPHOS networks (Supporting Information, Figure 2).

To identify the main important proteins within the discussed networks, we used the Cytoscape plug in "network analyzer" and a robustness test to identify the hubs. The main hub protein (in terms of centrality and robustness) in both networks was succinate dehydrogenase iron-sulfur subunit (SDHB; 2-fold down regulated due to HFD, 1.5-fold up regulated due to RGZ treatment), part of the TCA cycle and respiratory chain. Furthermore, succinyl-CoA ligase subunit alpha (SUCLG1; 1.5 fold down regulated for HFD and 1.5 fold up regulated for RGZ), catalyzing the ATP- or GTP-dependent ligation of succinate and $\mathrm{CoA}$ to form succinyl-CoA was a main hub (Table 2). This shows again the effectiveness of RGZ to turn HFD WAT into a LFD-like condition.

For a more detailed pathway analysis of transcripts, proteins, and metabolites, we picked the citric acid cycle (TCA) as a strongly regulated pathway between LFD/HFD condition in WAT (Figure 7). More than half of the detected RNAs were at least 1.5 -fold up regulated in the LFD compared with the HFD state, whereas all detected proteins in this pathway were not significantly regulated on the basis of individual protein ratios, although the whole protein pathway was significantly up regulated (see Supporting Information, Figure 1B). Notably, although RNA and protein levels were up regulated, metabolites within the TCA cycle were down regulated, except incoming acetyl-CoA, which was 2-fold up regulated. This result shows fine-tuned regulations at the three investigated interconnected biological levels: Up regulated levels of RNAs or proteins do not necessarily mean that there are more metabolites present, but may rather indicate compensatory relative effects to regain metabolic homeostasis.

We further analyzed the valine, leucine, and isoleucine degradation pathway in more detail between LFD/HFD condition in WAT (Figure 8). We observed a significant up regulation on the BCAA pathway level for RNA, protein, and metabolite data sets in adipose tissue (Supporting Information, Figure 1B). Notably, a recent study of morbidly obese subjects with type 2 diabetes suggested that the reduction of BCAAs in blood resulting from gastric bypass surgery may be associated with improvement in blood sugar regulation. ${ }^{41}$ Our WAT data indicate that increased amounts of BCAAs induce an increasing expression of genes and proteins required for degradation of BCAA, which might have an impact on levels of BCAAs in blood. Furthermore, the up regulation of this pathway in LFD compared to HFD could be a compensatory reaction, as this amino acid breakdown generates acyl-CoA derivatives, which can enter the TCA cycle. 

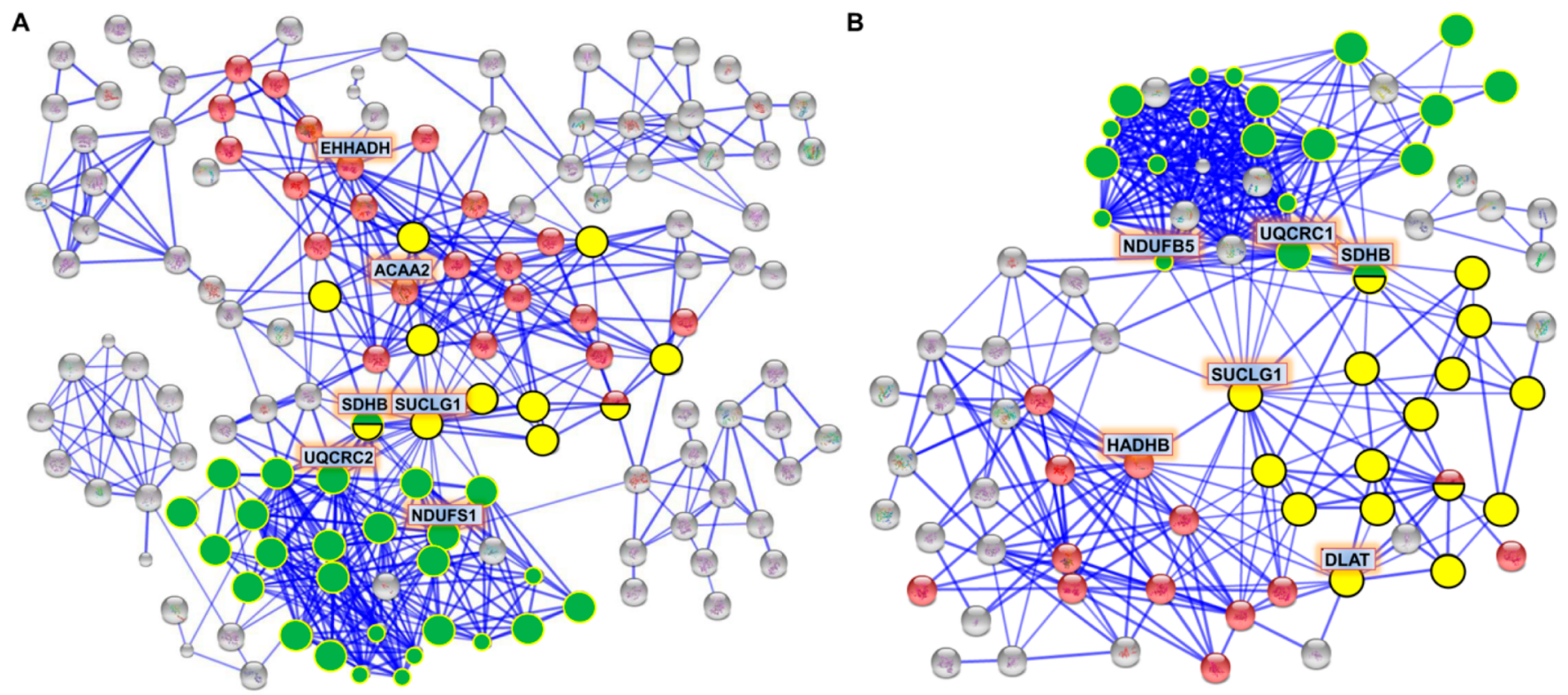

Figure 5. Network of down regulated proteins in (A) HFD and up regulated in (B) RGZ-HFD fat tissue, visualized by STRING software (version 9.1). (A) LFD/HFD ratios. (B) RGZ-HFD/HFD ratios. The top hub proteins are indicated according to Table 2. Proteins are colored according their KEGG pathway affiliation: red $=\mathrm{BCAA}$, yellow $=$ TCA cycle, and green $=$ OXPHOS .

Table 2. Network Analysis of Down Regulated HFD and Up Regulated RGZ-HFD Proteins in WAT To Determine the Main Hubs in Terms of Robustness and Betweenness Centrality ${ }^{a}$

\begin{tabular}{|c|c|c|c|c|c|c|c|}
\hline & protein & nodes & average distance & network diameter & node degree & betweenness centrality & closeness centrality \\
\hline \multirow[t]{7}{*}{ network down } & LFD/HFD & 165 & 4.49 & 12 & & & \\
\hline & SDHB & 164 & 4.66 & 12 & 20 & 0.21 & 0.34 \\
\hline & SUCLG1 & 163 & 4.81 & 12 & 21 & 0.12 & 0.34 \\
\hline & NDUFS1 & 158 & 6.82 & 19 & 16 & 0.11 & 0.32 \\
\hline & EHHADH & 154 & 7.48 & 21 & 18 & 0.08 & 0.27 \\
\hline & ACAA2 & 152 & 7.87 & 21 & 18 & 0.05 & 0.32 \\
\hline & UQCRC2 & 148 & 9.16 & 24 & 28 & 0.06 & 0.33 \\
\hline \multirow[t]{7}{*}{ network down } & RGZ/HFD & 108 & 2.83 & 8 & & & \\
\hline & SUCLG1 & 107 & 2.98 & 8 & 21 & 0.27 & 0.52 \\
\hline & UQCRC1 & 106 & 3.10 & 8 & 27 & 0.16 & 0.50 \\
\hline & NDUFB5 & 105 & 3.38 & 9 & 23 & 0.06 & 0.48 \\
\hline & SDHB & 104 & 3.52 & 9 & 16 & 0.04 & 0.43 \\
\hline & DLAT & 102 & 4.05 & 11 & 12 & 0.05 & 0.38 \\
\hline & HADHB & 100 & 4.46 & 11 & 19 & 0.12 & 0.45 \\
\hline
\end{tabular}

${ }^{a}$ Nodes are connected proteins within a network; the average distance between nodes is calculated after removal of a node; the diameter of a network is defined as the longest of all calculated shortest paths in a network. All three of these criteria are indicators of the network robustness and resilience. Node degree is the number of connections between one node to others; the betweenness centrality is equal to the number of shortest paths from all vertices to all others that pass through that node; closeness centrality is the distance metric between all nodes, defined by the length of their shortest paths. 